\title{
Augmented Th17-type immune responses in preterm neonates exposed to histologic chorioamnionitis
}

\author{
Daniel C. Rito' ${ }^{1}$ Luke T. Viehl' ${ }^{2}$ Paula M. Buchanan ${ }^{2,3}$, Seema Haridas ${ }^{2}$ and Joyce M. Koenig ${ }^{2,4}$
}

BACKGROUND: Histologic chorioamnionitis (HCA) is a placental inflammatory disorder that frequently precedes preterm delivery. HCA increases risk for long-standing inflammatory injury and may influence immune programming, particularly in preterm (PT) neonates. We hypothesized that HCA exposure is associated with an increased circulating frequency of proinflammatory, Th17-type responses.

METHODS: Placental cord blood was collected from HCAexposed or control neonates (23-41 wk gestation). Frequencies of Th17 and T regulatory (Treg) cells and assessments of Th17type features in CD4 and Treg cells were determined by flow cytometric analysis.

RESULTS: Cord blood samples from 31 PT and 17 term neonates were analyzed by flow cytometry. A diagnosis of HCA in extremely PT (EPT, GA $\leq 30$ wk) gestations was associated with the highest cord blood frequencies of progenitor (pTh17, $\left.\mathrm{CD}^{+}{ }^{\mathrm{C} D} 161^{+}\right)$and mature (mTh17, $\left.\mathrm{CD} 44^{+} \mathrm{CD} 161^{+} \mathrm{CCR} 6{ }^{+}\right)$Th17 cells. Preterm neonates exposed to HCA also exhibited elevated cord blood frequencies of $\mathrm{IL}-17^{+}$Treg cells, as well as T cells with effector memory phenotype (TEM) that coexpressed Th17-type surface antigens.

CONCLUSION: Th17-type responses are amplified in preterm neonates exposed to HCA. We speculate that a Th17 bias may potentiate the inflammatory responses and related morbidity observed in preterm neonates whose immune systems have been "primed" by HCA exposure.

$H$ istologic chorioamnionitis (HCA) is a neutrophil-driven inflammation of the placental membranes. A common antecedent of preterm delivery, the incidence and severity of HCA are inversely proportional to gestational age $(1,2)$. Extremely preterm neonates born after HCA exposure may also be at greater risk for chronic inflammatory disorders (3-7). However, the mechanisms leading to exaggerated inflammatory responses in these infants are incompletely understood. We recently reported that antenatal inflammation in a murine model induces exaggerated systemic inflammatory immune responses in exposed offspring, including enhanced lung expression of proinflammatory Th17 cells (3).
Th17 cells are a unique T helper subset that bridges adaptive and innate immune responses. While Th17 cells are critical to antimicrobial immunity, they can also mediate pathogenic inflammatory processes (reviewed in ref. (4)). Ontogenic studies suggest a skewing of CD4 cell differentiation towards Th17-type immunity in human preterm neonates and in the offspring of immune-stimulated pregnant mice $(3,5,6)$. Despite their importance to health and illness, however, the biology of Th17 cells in neonates and their role in inflammation remain incompletely defined.

We hypothesized that fetal exposure to inflammation during critical developmental windows can influence immune programming to augment inflammatory neonatal responses. To begin to test this, the goal of the present study was to determine Th17-type responses in preterm and term neonates exposed to intrauterine inflammation.

\section{METHODS}

Ethics Statement

This prospective observational study was performed with the approval of a protocol and according to the policies of the Institutional Review Board for Human Studies of Saint Louis University, SSM Cardinal Glennon Children's Hospital (CGCH), and SSM St. Mary's Health Center. Informed, written consent was obtained for all study participants.

\section{Human Subjects}

Study subjects and gestational age-matched controls consisted of neonates born to women admitted to the Labor \& Delivery service at SSM St. Mary's Health Center, a large perinatal referral center in St. Louis, MO, from January 2013 through June 2014. Women with intrauterine pregnancies of $23-41 \mathrm{wk}$ of gestation were prospectively enrolled in the study if they presented in preterm labor and/or had a clinical diagnosis of suspected chorioamnionitis. Potential subjects were excluded from study if mothers or pregnancies were affected by inflammatory conditions or infection other than suspected chorioamnionitis, or if a potential for altered immunity related to congenital or genetic conditions in the fetus or newborn existed. Demographic and clinical details were obtained from the electronic medical record.

\section{Diagnosis of Histologic Chorioamnionitis}

Placentas of all PT infants and of term infants with suspected clinical chorioamnionitis were examined by a clinical pathologist as part of routine clinical care. The diagnosis of histologic chorioamnionitis (HCA) has been typically defined by the extent of neutrophilic involvement of the chorionic plate, the subchorionic space, umbilical cord vessels, and/or Wharton's jelly $(8,9)$. The diagnosis of HCA was

\footnotetext{
'Department of Neonatology, Henry Ford Medical Group, Detroit, Michigan; ${ }^{2}$ Department of Pediatrics, Saint Louis University, St. Louis, Missouri; ${ }^{3}$ School of Public Health \& Social Justice, Saint Louis University, St. Louis, Missouri; ${ }^{4}$ Department of Molecular Microbiology \& Immunology, Saint Louis University, St. Louis, Missouri. Correspondence: Joyce M. Koenig (koenijm@slu.edu)

Received 1 June 2016; accepted 9 October 2016; advance online publication 1 February 2017. doi:10.1038/pr.2016.254
} 
based on placental findings consistent with a Stage 2 or higher maternal inflammatory response and/or any fetal inflammatory response (umbilical vasculitis, arteritis, or funisitis) (9). For the present study, gestations were diagnosed with HCA based on placental findings alone regardless of whether or not chorioamnionitis was clinically suspected. Preterm gestations were considered to be "no HCA" controls based on the absence of inflammatory infiltrates or their restriction to the chorionic plate, chorionic vessels, or subchorionic space (Stage 1 maternal inflammatory response). Gestations were also considered to be "no HCA" controls in the presence of presumptive clinical chorioamnionitis if placental pathology was not consistent with HCA.

\section{Cord Blood Collection}

Immediately following delivery, cord blood was drawn from the placental umbilical vein using antiseptic technique and collected into sterile syringes or cord blood collection bags containing anticoagulant. Cellular analyses of blood samples were performed within $12 \mathrm{~h}$ of collection.

\section{Antibodies and Reagents}

For multicolor flow cytometric analyses, the following fluorochromelabeled $\mathrm{Ab}$ and IgG subset controls (all purchased from BectonDickinson (BD, Franklin Lakes, NJ, unless otherwise indicated) were utilized for surface or intracellular staining of cells: CD3-Alexa Fluor 700 (clone UCHT1), CD3-PerCPCy5.5 (5k7), CD4-V450 (RPA-T4), CD4-PE (RPA-T4), CD4-FITC (RPA-T4), CD4-V500 (RPA-T4), CD25-BV605 (2A3), CD25-PE (2A3), CD127-PE (hIL-7R-M21), CD127-PerCPCy5.5 (hIL-7R-M21), CD196-BV605 (11A9), IL-17AAlexa Fluor 700 (N49-653), ROR $\gamma t-P E$ (Q21-555), FoxP3-V450 (259D/C7). Foxp3-APC (PCH101) was a component of the AntiHuman Foxp3 Staining Set (eBioscience, San Diego, CA). FacsLysis was purchased from BD. The following reagents were purchased from Sigma-Aldrich (St. Louis, MO): RPMI-1640, phosphate-buffered saline (PBS), fetal bovine serum, paraformaldehyde, phorbol 12-myristate 13-acetate, ionomycin, brefeldin A.

\section{Cell Preparation and Flow Cytometry}

To determine surface antigen expression in cord blood samples, whole blood (WB), or in some cases mononuclear cell fractions, were stained with optimized $\mathrm{mAb}$ concentrations followed by fixation with paraformaldehyde, as described (3). For intracellular cytokine detection, samples were first stimulated with PMA $(50 \mu \mathrm{g} / \mathrm{ml})$, ionomycin $(1 \mu \mathrm{g} / \mathrm{ml})$, and brefeldin $\mathrm{A}(5 \mu \mathrm{g} / \mathrm{ml})$ for $4 \mathrm{~h}$ at $37^{\circ} \mathrm{C}$ (or for unstimulated samples, incubated with PBS alone), followed by staining with $\mathrm{mAb}$ against surface antigens (CD4, CD25). After fixation and permeabilization procedures (Anti-Human Foxp3 Staining Set, eBioscience), cells were stained with fluorochrome-labeled $\mathrm{mAb}$ directed against intracellular antigens. For nuclear transcription factor studies without cytokine expression, samples were processed for intracellular staining without stimulation, as described. Type-specific, fluorochrome-labeled IgG controls for each mAb used were included in all surface or intracellular antigen studies, under stimulated or unstimulated conditions as appropriate. Stained and fixed replicate samples were acquired within $24 \mathrm{~h}$ of staining using a 16-color BD LSR II Flow Cytometer. Compensation settings were based on the analysis of samples with single color staining. Unstained samples were used to exclude intrinsic background fluorescence, which was minimal in all studies examined.

Acquired samples were analyzed using the FlowJo 7.2.2 software (Tree Star, Ashland, OR). Initial gates were set on the lymphocyte population using forward- and side-scatter characteristics, and specific lymphocyte subsets were identified within $\mathrm{CD}^{+} \mathrm{CD}^{+}$populations using fluorochrome-labeled $\mathrm{mAb}$ and IgG-subtype specific controls. In surface staining studies, Th17 cells were identified in $\mathrm{CD}^{+}$cells with surface expression of CD161 (pTh17, progenitor populations (10) or that were $\mathrm{CD} 161^{+} \mathrm{CCR}^{+}$(mTh17, mature populations (11). Within the gated $\mathrm{CD} 4^{+}$population Tregs were identified by $\mathrm{CD} 25^{\text {hi }} \mathrm{CD} 127^{\text {lo }}$ expression, and $\mathrm{T}$ effector memory cells (TEM) were identified by $\mathrm{CD} 25^{\mathrm{lo}} \mathrm{CD} 127^{\mathrm{hi}}$ expression (12). For intracellular antigen analyses studies of gated CD4 cells, Th17 cells were identified in populations that were ROR $\gamma t^{+} \mathrm{IL}_{-}$ $17 \mathrm{~A}^{+}$while Treg cells were identified by the CD $25^{\text {hi }} \mathrm{Foxp} 3^{+}$population. Specific gating strategies are summarized in Supplementary Data online (Supplementary Figures S1 and S2 online).

\section{Determination of Absolute Cell Numbers}

Complete blood counts were obtained on study subjects at the discretion of the medical team. Absolute lymphocyte numbers were calculated from the corrected white blood cell count only if a CBC had been obtained in the first $6 \mathrm{~h}$ of life. The proportions of CD4 cells and their subsets as determined by flow cytometry were used to calculate absolute cell numbers.

\section{Statistical Analysis}

Experimental data were compared using the Mann-Whitney rank test or the unpaired Student's $t$-test as appropriate, using Prism v6.03 (GraphPad Software, La Jolla, CA). A $P$ value $\leq 0.05$ was considered statistically significant. For demographic analyses, nominal variables are reported as frequencies, continuous variables are reported as means $\pm \mathrm{SD}$ (parametric) or as medians (25th-75th percentiles; nonparametric). Nominal variables were compared using Chi-square analysis or Fisher's exact test using PASW (SPSS, v23.0; IBM, Armonk, NY).

\section{RESULTS}

\section{Patient Characteristics/Demographics}

Forty-eight cord blood samples were variously analyzed by multi-parameter flow cytometry: 31 preterm (PT: 17 exposed to HCA, 14 unaffected controls, Ctrl) and 17 term (7 HCA, 10

Table 1. Gestational and perinatal demographics.

\begin{tabular}{lccccc}
\hline & PT-Ctrl $(n=14)$ & PT-HCA $(n=17)$ & $\boldsymbol{P}$ & T-Ctrl $(n=10)$ & T-HCA $(n=7)$ \\
\hline Maternal age (years) & $25.0 \pm 10.0$ & $26.0 \pm 8.0$ & NS & $25.5 \pm 14.0$ & $20.0 \pm 12.0$ \\
BMI & $28.3 \pm 9.4$ & $30.7 \pm 11.5$ & NS & $32.7 \pm 4.5$ & $30.9 \pm 8.8$ \\
Anesthesia & & NS & & NS \\
$\quad$ General/IV & $3(21 \%)$ & $4(24 \%)$ & NS & $1(10 \%)$ & $1(14 \%)$ \\
$\quad$ Spinal/Epidural & $7(50 \%)$ & $10(58 \%)$ & NS & $9(90 \%)$ & $6(86 \%)$ \\
$\quad$ Local/None & $4(29 \%)$ & $3(18 \%)$ & NS & 0 & 0 \\
GBS+ & $3(21 \%)$ & $3(18 \%)$ & NS & 0 & $1(14 \%)$ \\
Antenatal steroids & $14(100 \%)$ & $17(100 \%)$ & NS & 0 & 0 \\
PROM & $3(21 \%)$ & $13(77 \%)$ & $<0.01$ & $1(10 \%)$ & NS \\
Clinical chorioamnionitis & $1(7 \%)$ & $5(29 \%)$ & NS & $2(20 \%)$ & NS \\
GA & $29.4 \pm 2.5$ & $29.2 \pm 3.6$ & NS & $38.0 \pm 0.94$ & NS \\
BW & $1,213 \pm 274$ & $1,523 \pm 738$ & NS & $3,217 \pm 435$ & $40.0 \pm 0.82$ \\
\hline
\end{tabular}

GBS, group B Streptococcus antenatal screening culture; PROM, prolonged rupture of membranes $>18 \mathrm{~h}, \mathrm{GA}$ (weeks), BW (grams). Data are shown as mean \pm SEM. 
a

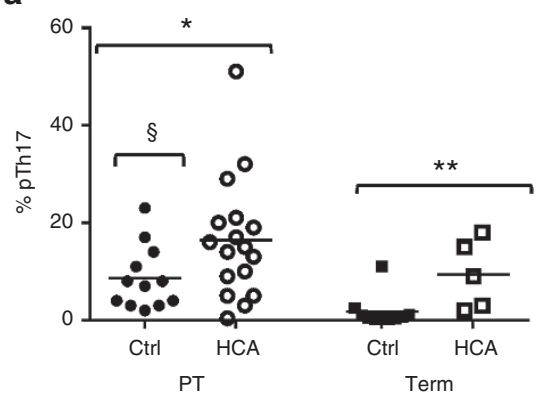

b

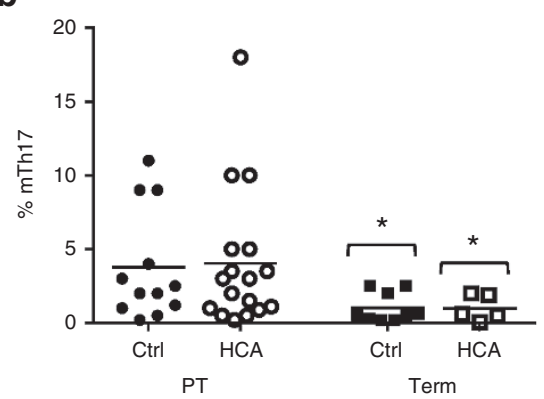

C

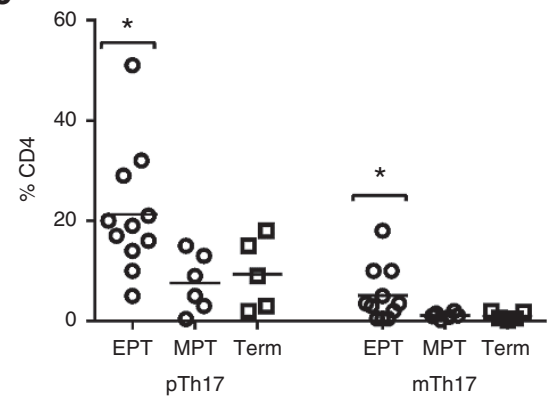

Figure 1. Gestational age and HCA effects on Th17 populations. Cord blood samples of control (Ctrl) or HCA-exposed PT ( $n=29)$ and term ( $n=12)$ neonates were analyzed by flow cytometry to determine the frequencies of Th17 cell subsets within gated $\mathrm{CD} 3^{+} \mathrm{CD} 4^{+}$cells $(\mathbf{a}-\mathbf{c})$. For the scatter plots with means presented here and in subsequent Figures, each data point represents the average value of replicate studies for an individual subject. (a) Frequencies of Th17 progenitor cells (pTh17, CD161+). ${ }^{*} P<0.05$, PT Ctrl vs. PT HCA; ${ }^{* *} P<0.01$, Term Ctrl vs. HCA; $\$ P<0.001$, PT Ctrl vs. Term Ctrl. (b) Frequencies of mature Th17 cells $\left(\mathrm{mTh} 17, \mathrm{CD} 161^{+} \mathrm{CCR} 6^{+}\right)$. ${ }^{*} \mathrm{P}<0.05$, Term vs. PT. c. Comparative frequencies of pTh17 and mTh17 cell populations in PT subsets (EPT $\leq 30$ wk GA; MPT, moderately PT, 31-34 wk GA) or in term neonates exposed to HCA. *P < 0.05, EPT vs. Term (pTh17); EPT vs. MPT, Term (mTh17).

Ctrl) (Table 1). The incidence of prolonged membrane rupture (PROM, $>18 \mathrm{~h}$ prior to delivery) was more common in gestations complicated by HCA for either gestational age group, consistent with previous reports $(7,13)$. All women enrolled in this study with impending preterm delivery received antenatal steroids. Gestational and perinatal characteristics were generally similar for HCA-exposed neonates and their age-matched controls. In PT gestations with a diagnosis of HCA, only 5 of 17 (29\%) of pregnant women had symptoms suggestive of clinical chorioamnionitis, while all term gestations with HCA had presumptive clinical chorioamnionitis.

\section{Th17 cell populations}

Higher frequencies of progenitor Th17 cells ( $\mathrm{pTh} 17, \mathrm{CD} 161^{+}$) within gated $\mathrm{CD}^{+}$populations were found in the cord blood of PT vs. term control neonates (Figure 1a). In both HCAexposed PT and term neonates, pTh17 frequencies were elevated relative to age-matched controls. The absolute numbers of circulating pTh17 cells were also higher in HCA-exposed PT neonates relative to PT controls (Table 2) and also as compared against HCA-exposed term neonates (PT, $0.71 \pm 0.14$ vs. Term, $\left.0.42 \pm 0.21 \times 10^{9} / 1 ; P<0.05 ; \mathrm{X} \pm \mathrm{SEM}\right)$. Frequencies of mature Th17 cells ( $\left.\mathrm{mTh} 17, \mathrm{CD} 161^{+} \mathrm{CCR} 6^{+}\right)$in gated CD 4 cells were higher in control PT vs. term neonates. In contrast to our observations in $\mathrm{pTh} 17$ cells, HCA exposure was not associated with altered $\mathrm{mTh} 17$ cell frequencies for either the PT group as a whole or in term neonates (Figure 1b), and no differences were observed in the absolute numbers of $\mathrm{mTh} 17$ cells between control and HCA-exposed PT neonates (Table 2). However, comparative analyses of gestational age subsets of HCA-exposed neonates showed an inverse relationship between pTh17 and mTh17 cell populations and gestational age, with the highest frequencies observed in HCA-exposed EPT (extremely PT, GA $\leq 30 \mathrm{wk}$ ) compared to MPT (moderately PT, GA 31-34 wk) and term neonates (Figure 1c).

\section{Treg populations}

A higher proportion of cord blood $\mathrm{CD}^{+}$cells were identified as Tregs $\left(\mathrm{CD} 25^{\mathrm{hi}} \mathrm{CD} 127^{\mathrm{lo}}\right)$ in control and HCA-exposed PT vs.
Table 2. Circulating Th17 and Treg cell numbers in preterm cord blood

\begin{tabular}{|c|c|c|c|}
\hline & \multicolumn{3}{|c|}{ Preterm } \\
\hline & Controls $(n)$ & $\mathbf{H C A}(n)$ & $\begin{array}{c}\text { P value } \\
\text { Ctrl vs. } \\
\text { HCA }\end{array}$ \\
\hline Lymphocytes $\left(\times 10^{9} / \mathrm{l}\right)$ & $4.13 \pm 0.40(14)$ & $5.11 \pm 0.54(17)$ & NS \\
\hline CD4\% $\left(\times 10^{9} / \mathrm{I}\right)$ & $64.6 \pm 2.7(13)$ & $67.8 \pm 2.4(17)$ & NS \\
\hline $\operatorname{CD4}\left(\times 10^{9} / \mathrm{l}\right)$ & $2.67 \pm 0.34(13)$ & $3.32 \pm 0.39(17)$ & NS \\
\hline pTh17 $\left(\times 10^{9} / \mathrm{l}\right)$ & $0.24 \pm 0.08(12)$ & $0.71 \pm 0.14^{*}(17)$ & $<0.05$ \\
\hline mTh17 (× 109/l) & $0.15 \pm 0.05(12)$ & $0.20 \pm 0.08(17)$ & NS \\
\hline IL-17+ (× 109/I) & $0.29 \pm 0.14(6)$ & $1.38 \pm 0.41^{*}(7)$ & $<0.01$ \\
\hline $\operatorname{Treg}\left(\times 10^{9} / \mathrm{I}\right)$ & $0.26 \pm 0.04(12)$ & $0.22 \pm 0.03(14)$ & NS \\
\hline Th17:Treg ratio & $0.59 \pm 0.19(12)$ & $4.25 \pm 0.95(14)$ & $<0.001$ \\
\hline
\end{tabular}

This Table summarizes Th17-type responses in CD4 lymphocyte subsets of preterm cord blood. The absolute lymphocyte count (ALC) was derived from the first postnatal CBC, if obtained within the first $6 \mathrm{~h}$ of life. Mean CD4 percentages represent the proportions in gated CD3+ lymphocyte populations. Mean percentages of Th17 and IL-17+ subsets represent their proportions within gated CD4 populations. Data are shown as mean \pm SEM.

${ }^{*} P<0.05$, PT HCA vs. Term HCA.

term neonates (Figure 2a). A diagnosis of HCA was associated with lower Treg proportions in $\mathrm{CD}^{+}$cells in the PT group, while a nonsignificant trend toward lower Treg frequencies was observed in term HCA-exposed vs. control neonates $(P=0.09)$. Comparative analyses of $\mathrm{CD}^{+}$cells in the cord blood of PT subsets and term populations revealed a trend of decreasing Treg cell frequencies with advancing gestational age (Figure 2b). However, although Treg frequencies within CD4 populations differed between control and HCA-exposed PT neonates, absolute Treg numbers in the cord blood were similar between these groups (Table 2).

\section{Th17:Treg ratio}

The circulating Th17:Treg ratio has been reported to correlate with disease severity in adult inflammatory disorders (reviewed in ref. (14)). For the present study, we calculated this ratio using frequencies of mTh17 or pTh17 cells and Treg 


\section{Articles | Ritoetal.}

cells in cord blood $\mathrm{CD}^{+}$populations of PT and term neonates. In comparative analyses, elevated mTh17:Treg ratios were observed in HCA-exposed EPT ( $\leq 30 \mathrm{wk}$ ) neonates relative to EPT controls, while no differences in this ratio were observed between HCA-exposed and control term neonates (Figure 2c). In contrast, the mean pTh17:Treg ratio, while generally higher in EPT vs. term neonates, did not appear to be affected by HCA exposure for either gestational group (Figure 2d). In contrast, when absolute numbers of pTh17 and Treg cells were used for calculations, an over sevenfold elevation in the pTh17:Treg ratio was observed in HCA-exposed PT neonates compared to controls (Table 2).

\section{TEM populations}

The TEM phenotype has been recently described in a subset of neonatal CD4 cells (12). To examine a potential effect of HCA on this cell population, we determined the frequencies of CD4 cells with a $\mathrm{CD} 25^{\mathrm{lo}} \mathrm{CD} 127^{\mathrm{hi}}$ phenotype. Frequencies of TEM a

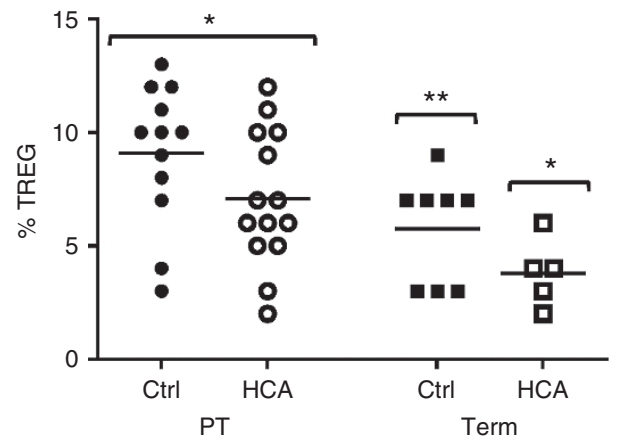

C

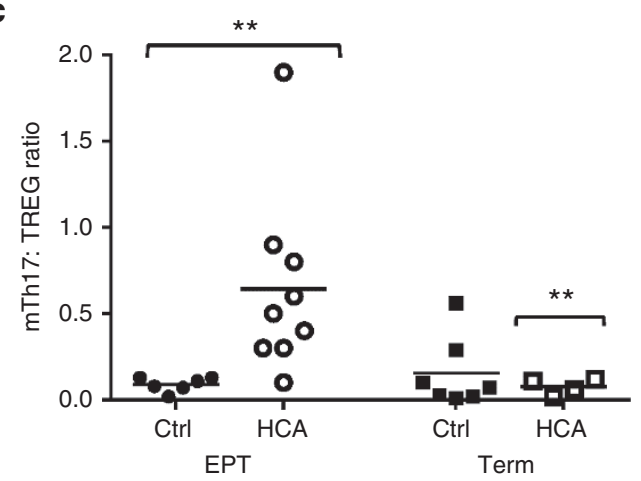

b

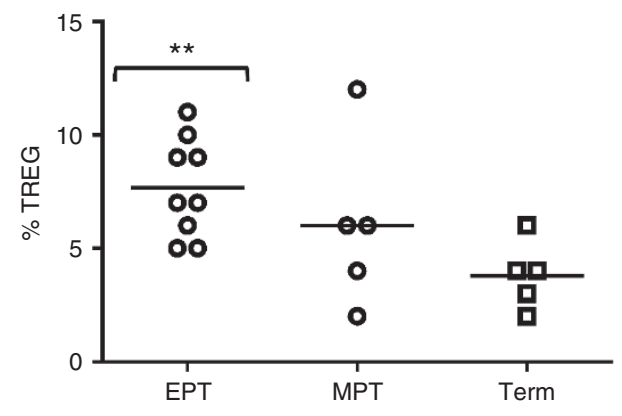

d

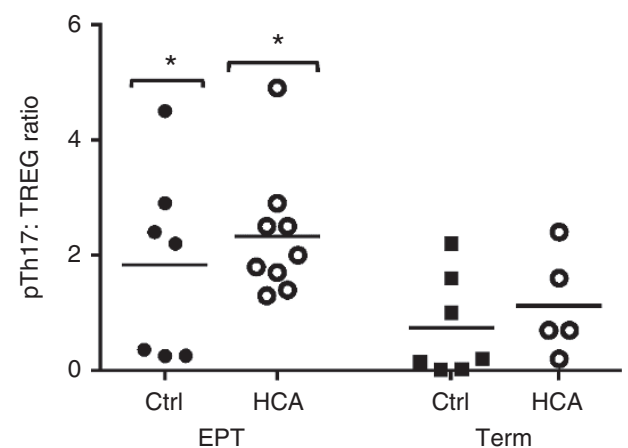

Figure 2. Gestational age and HCA influences Treg cell populations. Cord blood Treg cells $\left(C D 25^{\text {hi }} C D 127^{10}\right)$ within $C D 3^{+} C D 4^{+}$gated populations were identified by flow cytometry $(\mathbf{a}-\mathbf{d})$. The scatter plot graphs shown represent the proportions of Treg cells in gated CD4 ${ }^{+}$cells of PT and term neonates. (a) Frequencies of Treg cells in Ctrl and HCA-exposed PT $(n=28)$ and term $(n=13)$ neonates. ${ }^{*} P<0.05$, PT (Ctrl vs. HCA); Term HCA vs. PT HCA. ${ }^{* *} P<0.01$, Term Ctrl vs. PT Ctrl). (b) Comparative Treg frequencies in HCA-exposed EPT ( $\leq 30$ wk), MPT (31-34 wk), and term neonates. ${ }^{* *} P<0.01$, EPT vs. Term. (c,d) Comparative mTh17:Treg $(\mathbf{c})$ and pTh17:Treg $(\mathbf{d})$ ratios in EPT $(n=16)$ and term $(n=12)$ neonates. ${ }^{*} P<0.05$, EPT vs. Term. ${ }^{* *} P<0.01$, EPT (Ctrl vs. HCA); Term HCA vs. EPT HCA.

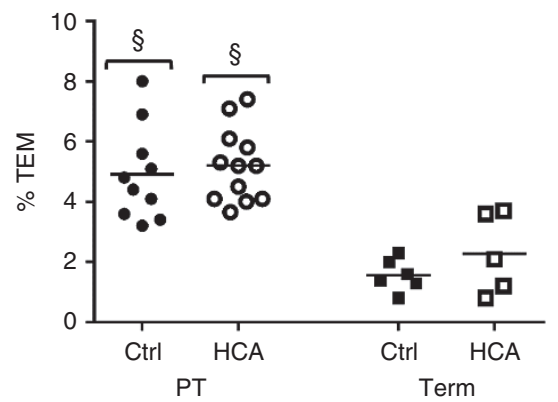

b

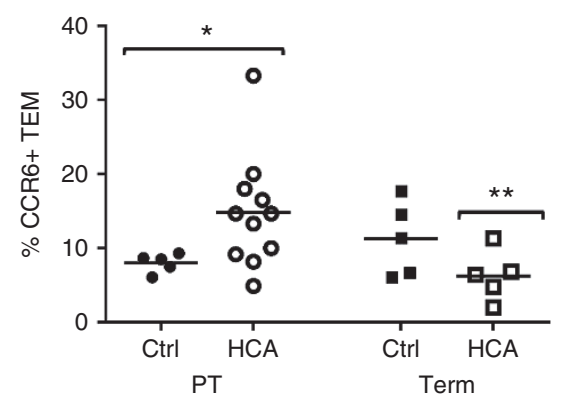

C

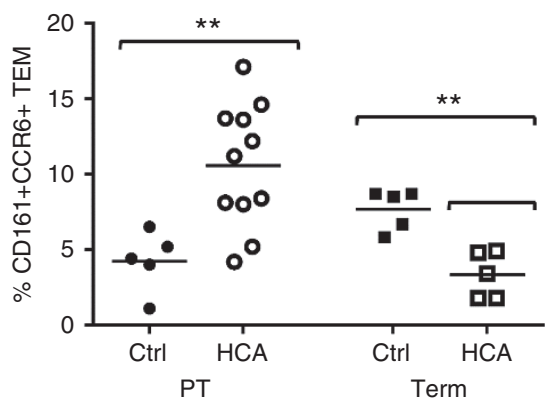

Figure 3. Effect of gestational age and HCA on TEM cells. TEM cells $\left(C D 25^{\circ} \mathrm{CD} 127^{\text {hi }}\right)$ were identified within $\mathrm{CD}^{+} \mathrm{CD} 4^{+}$gated populations in cord blood samples of PT ( $n=16-22)$ and term $(n=10-16)$ neonates. The scatter plot graphs shown represent the frequencies of TEM cells in cord blood CD4 ${ }^{+}$ populations of PT and term neonates (a), or the proportions of specific cell subsets within the TEM gate (b, $\mathbf{c})$ for each gestational age group. (a) TEM cell frequencies in $C D 4^{+}$cells. $\$ P<0.001$, PT vs. Term. (b) Frequencies of CCR6 ${ }^{+}$cells in TEM populations. ${ }^{*} P<0.05, P T\left(C t r l\right.$ vs. HCA). ${ }^{* *} P<0.01$, Term HCA vs. PT HCA. (c) Frequencies of $\mathrm{CD} 161^{+} \mathrm{CCR} 6^{+}$cells in TEM populations. ${ }^{* *} P<0.01$, Ctrl vs. HCA. 
cells were higher in PT relative to term controls, and exposure to HCA was not associated with altered proportions in either group (Figure 3a). In subset analyses, the frequencies of TEM cells that coexpressed CCR6 alone (Figure 3b) or in combination with CD161 (Figure 3c) were highest in PT HCA-exposed neonates. In contrast, whereas the mean expression patterns of the $\mathrm{CD}_{16} 1^{+} \mathrm{CCR}^{+}$subsets (Figure $3 \mathrm{c}$ ) were higher in HCAexposed $v s$. control $\mathrm{PT}$ neonates, this pattern was reversed in term subjects.

\section{IL-17A and RORyt Expression in CD4 Populations}

Cord blood studies in a small subset of neonates showed elevated frequencies of IL-17A $\mathrm{A}^{+}$(hence referred to as IL-17 $7^{+}$) CD4 cells in HCA-exposed PT and term neonates vs. agematched controls; frequencies of $\mathrm{IL}-17^{+} \mathrm{CD} 4$ cells were highest overall in the HCA-exposed PT group (Figure 4a). Similarly, absolute numbers of IL-17 ${ }^{+} \mathrm{CD} 4$ cells were higher in the cord blood of HCA-exposed PT neonates relative to PT controls (Table 2) or compared with HCA-exposed term neonates (Term, $0.63 \pm 0.31 \times 10^{9} / 1, \mathrm{P}<0.05 ; \mathrm{X} \pm \mathrm{SEM}$ ). Frequencies of CD4 cells that coexpressed both ROR $\gamma$ t and IL-17 were also elevated in HCA-exposed PT and term neonates relative to controls, and highest in PT HCA neonates (Figure 4b).
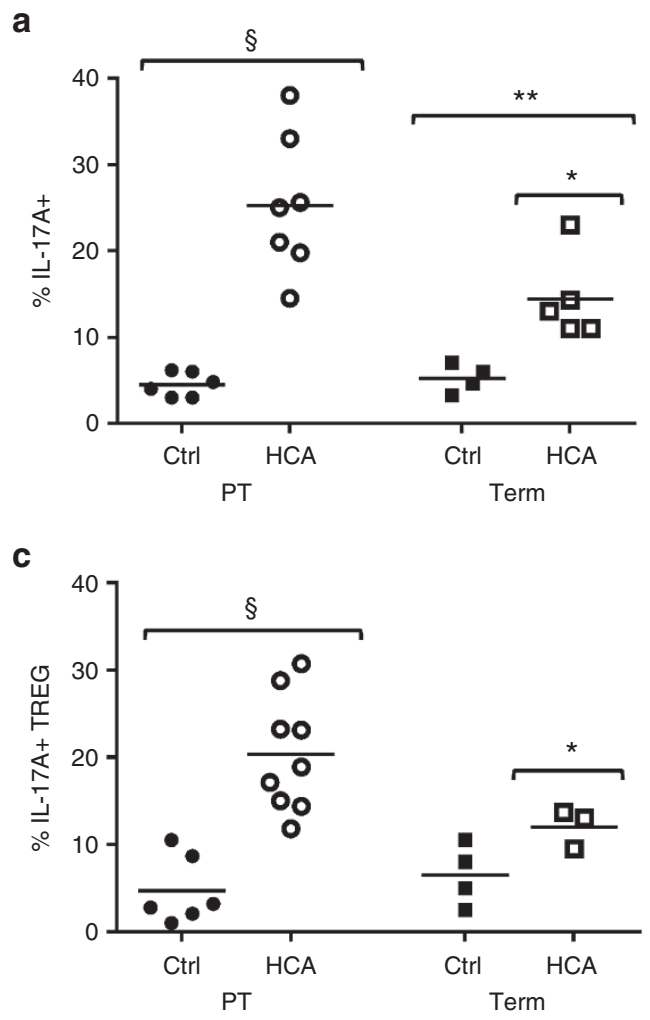

To determine if HCA exposure was associated with a Th17like phenotype in neonatal Treg cells, we next analyzed a small subset of $\mathrm{CD} 4^{+} \mathrm{C} 25^{\text {hi }}$ Foxp $3^{+}$Treg cells for intracellular expression of IL-17 or ROR $\gamma \mathrm{t}$. Mean proportions of IL- $17^{+}$ Tregs were quadrupled in PT-HCA neonates vs. PT controls; differences in the proportions of IL-17 ${ }^{+}$Tregs between HCA and control term neonates approached but did not reach significance $(P=0.06$; Figure $4 \mathrm{c})$. The proportions of ROR $\gamma \mathrm{t}^{+}$Treg cells were also higher in PT vs. term controls (Figure 4d). Although Treg coexpression of ROR $\gamma$ t was not different between control and HCA-exposed PT neonates, analyses of this small group of term neonates with HCA exposure showed increased proportions of ROR $\gamma \mathrm{t}^{+}$Tregs relative to age-matched controls.

\section{DISCUSSION}

The goal of the present study was to compare the potential effects of histologic chorioamnionitis (HCA) on the Th17 phenotype in PT and term neonates. We now report Th17-type responses in the cord blood of HCA-exposed neonates that appear to be particularly prominent in extremely PT neonates. To our knowledge, this is the first report of the prevalence of immune cells with Th17-like properties in human PT and term neonates in the context of HCA.

b

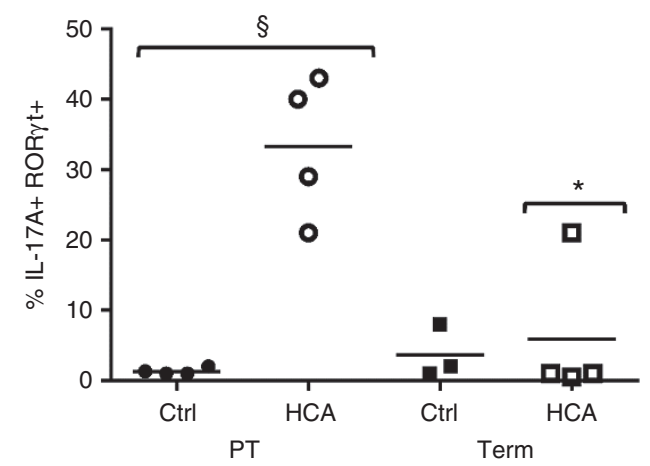

d

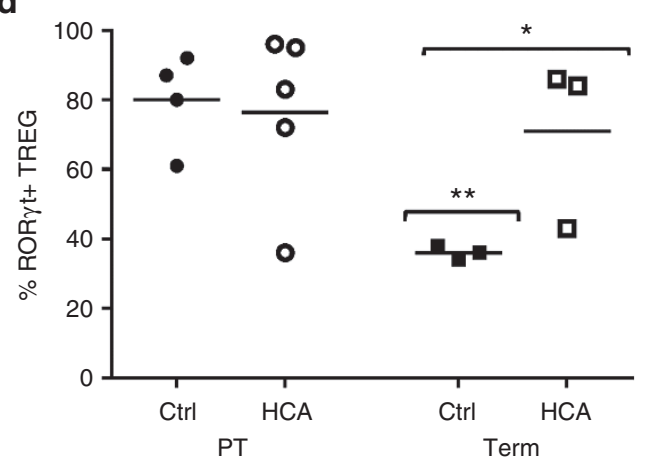

Figure 4. IL-17 expression in gated CD4 cells and Treg cells. The frequencies of cells that co-expressed IL-17A (IL-17) and/or RORyt within gated CD4 or Treg $\left(\mathrm{CD}^{+} \mathrm{CD}_{25}{ }^{+} \mathrm{FoxP3}^{+}\right)$populations were identified by flow cytometry in the cord blood of PT $(n=8-15)$ and term ( $\left.n=6-9\right)$ neonates with HCA or in Ctrl. Data are presented as scatter plots of the mean values from individual subjects (a-d). (a) Frequencies of IL- $17^{+}$cells in gated CD4 $4^{+}$populations. ${ }^{*} P<0.05$, Term HCA vs. PT HCA. ${ }^{* *} P<0.01$, Term (Ctrl vs. HCA). $§ P<0.001$, PT (Ctrl vs. HCA). (b) Frequencies of CD4+ cells coexpressing both IL-17 and the Th17 nuclear transcription factor, RORүt. ${ }^{*} P<0.05$, Term HCA vs. PT HCA. $§ P<0.001$, PT (Ctrl vs. HCA). (c) Frequencies of IL-17 ${ }^{+}$cells within gated Treg populations. ${ }^{*} P<0.05$, Term HCA vs. PT HCA. $\S P<0.001$, PT (Ctrl vs. HCA). d. Frequencies of ROR $\gamma \mathrm{t}^{+}$cells within Treg populations. ${ }^{*} P<0.05$, Term $(C$ trl vs. HCA). ${ }^{* * P}<0.01$, Term Ctrl vs. PT Ctrl. 


\section{Articles | Ritoetal.}

We observed relatively higher proportions of Th17 cell populations in PT relative to term neonates, consistent with the low Th17-type responses reported in normal term pregnancies (15). The higher levels of Th17 cells in HCA-exposed PT vs. term neonates suggest an influence of developmental stage, consistent with the intrinsic Th17 bias in PT neonates reported by Black et al. (5). Rueda et al. recently reported a similar elevation of CD4 ${ }^{+}$IL- $17^{+}$Th17 cells in the spleens of LPS-exposed fetal rhesus macaques (16). In the present study, we observed particularly robust frequencies of the $\mathrm{CD} 4^{+} \mathrm{CD} 161^{+}$(pTh17) cell subset in the cord blood of PT neonates, a finding which may have pathogenic implications for this population. Pertinently, high frequencies of $\mathrm{CD}_{161^{+}}$Th17 cells have been correlated with inflammatory disease activity and recent evidence indicates that this cell population is both excessively inflammatory and resistant to Treg-mediated suppression $(17,18)$. Higher coexpression of the canonical Th17 nuclear transcription factor, ROR $\gamma$ t, and IL-17 was also determined in cord blood CD4 cells of HCA-exposed PT neonates. Taken together, our findings parallel the elevated Th17 cell frequencies or Th17-type cytokine levels observed in human neonates exposed to maternal filarial infection, HIV, or pre-eclampsia (19-21) or in a nonhuman primate model of intrauterine inflammation (16). The increased proportions of Th17 cells in HCA-exposed neonates also likely reflect the heightened Th17-type responses in placentas with chorioamnionitis $(22,23)$.

Higher Treg frequencies were determined in the cord blood of PT relative to term control neonates, in keeping with previous reports (24). Although Treg proportions were decreased in HCA-exposed PT neonates relative to age-matched controls, an observation consistent with the findings of Treg depletion in a fetal nonhuman primate model of chorioamnionitis (16), the absolute numbers of Tregs were similar in PT groups. These data suggest that the elevation of absolute Th17 numbers in HCAexposed PT neonates occurs independently of an effect of HCA on Tregs. Given that elevation of the Th17:Treg ratio reflects disease severity in other Th17-associated inflammatory disorders (14), our findings suggest an enhanced Th17-mediated inflammatory response in PT neonates in the context of HCA.

Treg cells typically function to restrain activated $\mathrm{T}$ cells, including inflammatory Th17 cells $(14,25)$. However, Treg cells can also exhibit Th17-like phenotypes in the context of an inflammatory milieu (25). In the present study, we determined a subset of cord blood Treg cells with proinflammatory Th17-type characteristics in HCA-exposed neonates, a finding also reported in a fetal primate model of chorioamnionitis (16). Although the proportions of cord blood Treg cells that also coexpressed IL-17 were not different between PT and term controls, we observed that PT neonates exposed to HCA exhibited a particularly robust elevation of IL-17 ${ }^{+}$Tregs. In contrast, ROR $\gamma \mathrm{t}^{+}$ Treg frequencies were markedly higher in the cord blood of preterm vs. term control neonates, a finding that may reflect developmental regulation of ROR $\gamma \mathrm{t}$ and the common lineage shared by Tregs and Th17 cells $(5,26)$. Frequencies of ROR $\gamma t^{+}$ Tregs were not different between control and HCA-exposed PT neonates. In contrast, HCA exposure of term neonates was associated with higher $\mathrm{ROR} \gamma \mathrm{t}^{+}$Treg proportions relative to age-matched controls. Although Tregs with Th17-type features potentially retain suppressive activity (27), high ROR $\gamma$ t expression can contribute to loss of Treg anti-inflammatory function (28). In addition, ROR $\gamma t$ expression can facilitate the conversion of Tregs to a Th17 phenotype during inflammation $(28,29)$. Thus, the high ROR $\gamma t$ expression in Tregs of HCA-exposed PT and term neonates could contribute to compromised Treg suppressor function and the enhanced Th17-type responses that we now report. While recent data are supportive of this premise $(16,30)$, the effects of HCA exposure on Treg function in human neonates remain to be more fully elucidated.

Our studies included an analysis of cord blood $\mathrm{T}$ effector memory (TEM) cells, a recently characterized CD4 subset with a $\mathrm{CD} 25^{\mathrm{lo}} \mathrm{CD} 127^{\text {hi }}$ phenotype (12). Neonatal TEM express various Th17-related markers and produce IL-17 under inflammatory conditions (12). A higher proportion of TEM cells were observed in the cord blood of PT relative to term controls, and these were not altered with HCA exposure for either group. However, PT neonates exposed to HCA exhibited high frequencies of TEM subsets with Th17-type phenotypes (CD161 or CCR6 coexpression), observations that may also be relevant to amplified inflammatory function (19). While the clinical significance of these findings is at present unclear, the potential contribution of TEM cells to Th17-type inflammatory responses in PT neonates is deserving of further exploration.

The present study was not powered to identify infectious etiologies of HCA or to extrapolate experimental findings to HCA-associated neonatal outcomes $(20,21)$. In addition, only a limited number of gestations retrospectively met some, but not all, of the criteria needed for the newly-defined diagnosis of "confirmed Triple I" (intrauterine inflammation or infection or both) (31)), thus we were unable to employ this designation in the analyses of the present studies. However, our data confirmed a reported association between PROM and HCA $(7,13)$. Despite our limited sample size, the significant intergroup differences in Th17-type responses that we now report suggest biological relevance. However, it will be important to confirm and extend these findings in studies involving larger neonatal populations in order to establish clinical significance.

In summary, fetal exposure to HCA is associated with enhanced Th17-type responses in the cord blood of PT and term neonates. The prominence of the Th17 phenotype in PT neonates may be due to a combined intrinsic Th17 bias and a unique sensitivity of the developing immune system to environmental alterations (32). Our findings of enhanced Th17-type responses in addition to reports of diminished Treg suppressive activity $(16,30)$ suggest a compelling explanation for the potentiated inflammatory responses reported in HCA-exposed preterm neonates (3-6). While enhanced Th17 responses in preterm neonates could potentially augment protective immunity (4), as suggested by some evidence of decreased late-onset sepsis in gestations with chorioamnionitis (33), exposure to HCA has also been associated with increased neonatal susceptibility to infection (34). Furthermore, Wynn et al. recently showed a striking contribution of IL-17 signaling to inflammation-related neonatal mortality in a murine model of 
sepsis (35). Thus, new understanding of how antenatal inflammation both 'primes' the neonatal immune system to promote chronic inflammatory conditions and influences protective immune mechanisms will be critical to the development of effective treatment approaches for this fragile population.

\section{SUPPLEMENTARY MATERIAL}

Supplementary material is linked to the online version of the paper at http:// www.nature.com/pr

\section{ACKNOWLEDGMENTS}

We thank Guangyong Peng, David Randolph, and Noah Hillman for their critical reviews of our manuscript and helpful comments. We gratefully acknowledge the SSM St. Mary's Health Center Labor \& Delivery physicians and staff, and our neonatology colleagues, Mohamad Al-Hosni and Justin Josephsen, for their clinical assistance with our studies. We thank Ryan Weisert, Hong Wang, and Philip Petrosky for their expert technical skills, and thank Benjamin Schroeder for assisting with data analysis.

\section{STATEMENT OF FINANCIAL SUPPORT}

This work was supported in part by the Department of Pediatrics, Saint Louis University, St. Louis, MO; the Cardinal Glennon Foundation, St. Louis, MO; the Fleur de Lis Foundation, St. Louis, MO; and the National Institutes of Health (Al094478) to J.M.K.

Disclosure: The authors have no potential/perceived conflicts of interest.

\section{REFERENCES}

1. Gargano JW, Holzman C, Senagore P, et al. Mid-pregnancy circulating cytokine levels, histologic chorioamnionitis and spontaneous preterm birth. J Reprod Immunol 2008;79:100-10.

2. Lahra MM, Jeffery HE. A fetal response to chorioamnionitis is associated with early survival after preterm birth. Am J Obstet Gynecol 2004;190: $147-51$.

3. Gleditsch DD, Shornick LP, Van Steenwinckel J, Gressens P, Weisert RP, Koenig JM. Maternal inflammation modulates infant immune response patterns to viral lung challenge in a murine model. Pediatr Res 2014;76: 33-40.

4. McGeachy MJ, McSorley SJ. Microbial-induced Th17: superhero or supervillain? J Immunol 2012;189:3285-91.

5. Black A, Bhaumik S, Kirkman RL, Weaver CT, Randolph DA. Developmental regulation of Th17-cell capacity in human neonates. Eur J Immunol 2012;42:311-9.

6. Mandal M, Marzouk AC, Donnelly R, Ponzio NM. Preferential development of Th17 cells in offspring of immunostimulated pregnant mice. J Reprod Immunol 2010;87:97-100.

7. Romero R, Chaiworapongsa T, Alpay Savasan Z, et al. Damage-associated molecular patterns (DAMPs) in preterm labor with intact membranes and preterm PROM: a study of the alarmin HMGB1. J Matern Fetal Neonatal Med 2011;24:1444-55.

8. Redline RW. Inflammatory responses in the placenta and umbilical cord. Semin Fetal Neonatal Med 2006;11:296-301.

9. Redline RW. Inflammatory response in acute chorioamnionitis. Semin Fetal Neonatal Med 2012;17:20-5.

10. Cosmi L, De Palma R, Santarlasci V, et al. Human interleukin 17-producing cells originate from a CD161+CD4+ T cell precursor. J Exp Med 2008;205:1903-16.

11. Crome SQ, Clive B, Wang AY, et al. Inflammatory effects of ex vivo human Th17 cells are suppressed by regulatory T cells. J Immunol 2010;185: 3199-208.

12. Zhang X, Mozeleski B, Lemoine S, et al. 2014 CD4 T cells with effector memory phenotype and function develop in the sterile environment of the fetus. Sci Transl Med 6:238ra72.

13. Tambor V, Kacerovsky M, Lenco J, Bhat G, Menon R. Proteomics and bioinformatics analysis reveal underlying pathways of infection associated histologic chorioamnionitis in pPROM. Placenta 2013;34:155-61.
14. Noack M, Miossec P. Th17 and regulatory T cell balance in autoimmune and inflammatory diseases. Autoimmun Rev 2014;13:668-77.

15. Nakashima A, Ito M, Yoneda S, Shiozaki A, Hidaka T, Saito S. Circulating and decidual Th17 cell levels in healthy pregnancy. Am J Reprod Immunol 2010;63:104-9.

16. Rueda CM, Presicce P, Jackson CM, et al. Lipopolysaccharide-induced chorioamnionitis promotes IL-1-dependent inflammatory FOXP3+ CD4+ T cells in the fetal rhesus macaque. J Immunol 2016;196:3706-15.

17. Miao J, Zhang K, Lv M, et al. Circulating Th17 and Th1 cells expressing CD161 are associated with disease activity in rheumatoid arthritis. Scand J Rheumatol 2014;43:194-201.

18. Basdeo SA, Moran B, Cluxton D, et al. Polyfunctional, pathogenic CD161+ Th17 lineage cells are resistant to regulatory T cell-mediated suppression in the context of autoimmunity. J Immunol 2015;195:528-40.

19. Maggi L, Santarlasci V, Capone M, et al. CD161 is a marker of all human IL-17-producing T-cell subsets and is induced by RORC. Eur J Immunol 2010;40:2174-81.

20. Hecht JL, Fichorova RN, Tang VF, Allred EN, McElrath TF, Leviton A; Elgan Study Investigators. Relationship between neonatal blood protein concentrations and placenta histologic characteristics in extremely low GA newborns. Pediatr Res 2011;69:68-73.

21. Dando SJ, Nitsos I, Kallapur SG, et al. The role of the multiple banded antigen of Ureaplasma parvum in intra-amniotic infection: major virulence factor or decoy? PLoS One 2012; 7:e29856.

22. Kallapur SG, Presicce P, Senthamaraikannan P, et al. Intra-amniotic IL-1 $\beta$ induces fetal inflammation in rhesus monkeys and alters the regulatory $\mathrm{T}$ cell/IL-17 balance. J Immunol 2013;191:1102-9.

23. Ito $\mathrm{M}$, Nakashima A, Hidaka T, et al. A role for IL-17 in induction of an inflammation at the fetomaternal interface in preterm labour. J Reprod Immunol 2010;84:75-85.

24. Luciano AA, Arbona-Ramirez IM, Ruiz R, et al. Alterations in regulatory T cell subpopulations seen in preterm infants. PLoS One 2014;9:e95867.

25. Crome SQ, Wang AY, Levings MK. Translational mini-review series on Th17 cells: function and regulation of human T helper 17 cells in health and disease. Clin Exp Immunol 2010;159:109-19.

26. Weaver CT, Hatton RD. Interplay between the TH17 and TReg cell lineages: a (co-)evolutionary perspective. Nat Rev Immunol 2009;9:883-9.

27. Wang $\mathrm{T}$, Sun $\mathrm{X}$, Zhao J, et al. Regulatory $\mathrm{T}$ cells in rheumatoid arthritis showed increased plasticity toward Th17 but retained suppressive function in peripheral blood. Ann Rheum Dis 2015;74:1293-301.

28. Blatner NR, Mulcahy MF, Dennis KL, et al. Expression of RORyt marks a pathogenic regulatory $\mathrm{T}$ cell subset in human colon cancer. Sci Transl Med 2012;4:164ra159.

29. Yang XO, Nurieva R, Martinez GJ, et al. Molecular antagonism and plasticity of regulatory and inflammatory $\mathrm{T}$ cell programs. Immunity 2008;29: $44-56$.

30. Rueda CM, Wells CB, Gisslen T, Jobe AH, Kallapur SG, Chougnet CA. Effect of chorioamnionitis on regulatory $\mathrm{T}$ cells in moderate/late preterm neonates. Hum Immunol 2015;76:65-73.

31. Higgins RD, Saade G, Polin RA, et al.; Chorioamnionitis Workshop Participants. Evaluation and management of women and newborns with a maternal diagnosis of chorioamnionitis: summary of a workshop. Obstet Gynecol 2016;127:426-36.

32. Prescott SL. Early-life environmental determinants of allergic diseases and the wider pandemic of inflammatory noncommunicable diseases. J Allergy Clin Immunol 2013;131:23-30.

33. Strunk T, Doherty D, Jacques A, et al. Histologic chorioamnionitis is associated with reduced risk of late-onset sepsis in preterm infants. Pediatrics 2012;129:e134-41.

34. Wolfs TG, Jellema RK, Turrisi G, Becucci E, Buonocore G, Kramer BW. Inflammation-induced immune suppression of the fetus: a potential link between chorioamnionitis and postnatal early onset sepsis. J Matern Fetal Neonatal Med 2012;25 Suppl 1:8-11.

35. Wynn JL, Wilson CS, Hawiger J, et al. Targeting IL-17A attenuates neonatal sepsis mortality induced by IL-18. Proc Natl Acad Sci USA 2016;113:E2627-35. 